\section{Forschende \\ Komplementärmedizin}

Wissenschaft • Praxis • Perspektiven

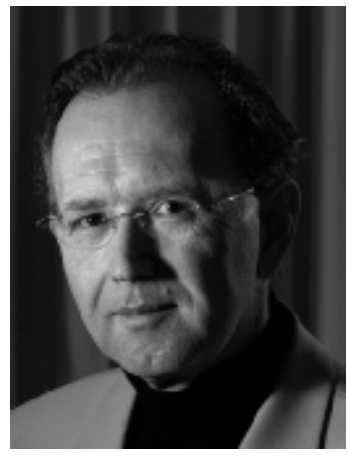

\title{
Latest Publications You Should Not Miss
}

\author{
Selected by \\ Dieter Melchart \\ München
}

Sacks N, Cabral H, Kazis LE, Jarrett KM, Vetter D, Richmond R, Moore TJ: A web-based nutrition program reduces health care costs in employees with cardiac risk factors: before and after cost analysis. J Med Internet Res 2009;11(4):e43(doi:10.2196/jmir.1263).

Background: Rising health insurance premiums represent a rapidly increasing burden on employer-sponsors of health insurance and their employees. Some employers have become proactive in managing health care costs by providing tools to encourage employees to directly manage their health and prevent disease. One example of such a tool is DASH for Health, an Internet-based nutrition and exercise behavior modification program. This program was offered as a free, opt-in benefit to US-based employees of the EMC Corporation.

Objective: The aim was to determine whether an employer-sponsored, Internet-based diet and exercise program has an effect on health care costs.

Methods: There were 15,237 total employees and spouses who were included in our analyses, of whom 1,967 enrolled in the DASH for Health program (DASH participants). Using a retrospective, quasi-experimental design, study year health care costs among DASH participants and nonparticipants were compared, controlling for baseline year costs, risk, and demographic variables. The relationship between how often a subject visited the DASH website and health care costs also was examined. These relationships were examined among all study subjects and among a subgroup of 735 subjects with cardiovascular conditions (diabetes, hypertension, hyperlipidemia). Multiple linear regression analysis examined the relationship of program use to health care costs, comparing study year costs among DASH participants and non-participants and then examining the effects of increased website use on health care costs. Analyses were repeated among the cardiovascular condition subgroups.

Results: Overall, program use was not associated with changes in health care costs. However, among the cardiovascular risk study subjects, health care costs were US\$ 827 lower, on average, during the study year $(\mathrm{p}=0.05 ; \mathrm{t} 729=1.95)$. Among 1,028 program users, increased website use was significantly associated with lower health care costs among those who visited the website at least nine times during the study year (US\$14 decrease per visit; $\mathrm{p}=0.04 ; \mathrm{t} 1022=2.05)$, with annual savings highest among 80 program users with targeted conditions (US\$ 55 decrease per visit; $\mathrm{p}<0.001 ; \mathrm{t} 74=2.71$ ).

Conclusions: An employer-sponsored, Internet-based diet and exercise program shows promise as a low-cost benefit that contributes to lower health care costs among persons at higher risk for above-average health care costs and utilization.

\section{Bausell RB: Are positive alternative medical therapy trials credible? Evidence from four high-impact medical journals. Evaluation Health Professions 2009; doi: $10.1177 / 0163278709346810$.}

Forty-five complementary and alternative medicine (CAM) efficacy randomized controlled trials (RCTs) from high-impact medical journals (NEJM, JAMA, Annals of Internal Medicine, and Archives of Internal Medicine) were reviewed based on their meeting three validity criteria (the existence of a placebo control, moderate attrition rates, and 50 or more participants per group). Of the 26 efficacy trials meeting all three criteria, only $2(7.7 \%)$ were judged to be positive (i.e., the alternative therapy was significantly superior to its placebo control), while over half (55.5\%) of the 19 trials that failed to meet one or more of these criteria reported positive results $(\mathrm{p}<0.001)$. Of the two positive high-validity trials, one was funded and authored by the herbal company marketing the product tested and one used a placebo-control group of questionable credibility. This analysis is consistent with the hypothesis that CAM therapies are no more effective than placebos when adequate experimental control is present

\section{Manheimer E, Wieland S, Kimbrough E, Cheng K, Berman BM: Evidence from the Cochrane Collaboration for traditional Chinese medicine therapies. J Altern Complement Med 2009;15(9):1001-1014.}

Background: The Cochrane Collaboration, an international not-for-profit organization that prepares and maintains systematic reviews of rando-

\section{KARGER}

Fax +497614520714

Information@Karger.de

www.karger.com 
mized trials of health care therapies, has produced reviews summarizing much of the evidence on Traditional Chinese Medicine (TCM). Our objective was to review the evidence base according to Cochrane systematic reviews.

Methods: In order to detect reviews focusing on TCM, we searched the titles and abstracts of all reviews in Issue 4, 2008 of the Cochrane Database of Systematic Reviews. For each review, we extracted data on the number of trials included and the total number of participants. We provided an indication of the strength of the review findings by assessing the reviewers" abstract conclusions statement. We supplemented our assessment of the abstract conclusions statements with a listing of the comparisons and outcomes showing statistically significant meta-analyses results.

Results: We identified 70 Cochrane systematic reviews of TCM, primarily acupuncture $(\mathrm{n}=26)$ and Chinese herbal medicine $(\mathrm{n}=42)$, and 1 each of moxibustion and t'ai chi. Nineteen (19) of 26 acupuncture reviews and 22/42 herbal medicine reviews concluded that there was not enough good quality trial evidence to make any conclusion about the efficacy of the evaluated treatment, while the remaining 7 acupuncture and 20 herbal medicine reviews and each of the moxibustion and t'ai chi reviews indicated a suggestion of benefit, which was qualified by a caveat about the poor quality and quantity of studies. Most reviews included many distinct interventions, controls, outcomes, and populations, and a large number of different comparisons were made, each with a distinct forest plot.

Conclusions: Most Cochrane systematic reviews of TCM are inconclusive, due specifically to the poor methodology and heterogeneity of the studies reviewed. Some systematic reviews provide preliminary evidence of Chinese medicine's benefits to certain patient populations, underscoring the importance and appropriateness of further research. These preliminary findings should be considered tentative and need to be confirmed with rigorous randomized controlled trials.

\section{Rogozina OP, Bonorden MJL, Grande JP, Cleary MP: Serum insulin-like growth factor-I and mammary tumor development in ad libitum-fed, chronic calorie-restricted, and intermittent calorie-restricted MMTV-TGF- $\alpha$ mice. Cancer Prev Res 2009;2:712-719.}

The effect of chronic (CCR) and intermittent (ICR) caloric restriction on serum insulin-like growth factor (IGF)-I levels and mammary tumor (MT) development was investigated. Ten-week-old MMTV-TGF- $\alpha$ female mice were assigned to ad libitum-fed (AL; AIN-93M diet), ICR [3-week 50\% caloric restriction using AIN-93M-mod diet, 2 x protein, fat, vitamins, and minerals followed by 3 weeks of daily $100 \%$ AL consumption of AIN-93M ( $75 \%$ of AL for each 6-week cycle)], and CCR (calorie and nutrient intake matched for each 6-week ICR cycle) groups. Half of the mice from each group were sacrificed at 79 (end of restriction) or 82 (end of refeeding) weeks of age. Serum was obtained at euthanasia and in cycles $1,3,5,8$, and 11 . MT incidence was $71.0 \%, 35.4 \%$, and $9.1 \%$ for AL, CCR, and ICR mice. ICR-restricted mice had significantly lower terminal serum IGF-I and IGF-I/IGF binding protein-3 (IGFBP-3) ratio than CCR, ICR-refed, and AL mice. There were no differences in terminal IGFBP-3. Final body, internal, and mammary fat pad weights correlated positively with IGF-I and negatively with IGFBP-3. Few changes were found for protein expression of IGF-IR $\alpha$ and IGFBP- 3 in mammary tissue and MTs. During the study, IGF-I levels of ICR-restricted mice were reduced, whereas refeeding allowed partial recovery. For all groups, elevated IGF-I levels preceded MT detection, although not all values were significant versus mice without MTs. However, the specific role of IGF-I in the protective effect of calorie restriction remains to be determined. These results confirm that ICR prevents MT development to a greater extent than CCR.

\section{Thaler K, Kaminski A, Chapman A, Langley T, Gartlehner G: Bach Flower Remedies for psychological problems and pain: a systematic review. BMC Complement Altern Med 2009,9:16.}

Background: Bach Flower Remedies are thought to help balance emotional state and are commonly recommended by practitioners for psychological problems and pain. We assessed whether Bach Flower Remedies (BFRs) are safe and efficacious for these indications by performing a systematic review of the literature.

Methods: We searched MEDLINE ${ }^{\oplus}$, Embase, AMED, and the Cochrane Library from inception until June 2008 and performed a handsearch of references from relevant key articles. For efficacy, we included all prospective studies with a control group. For safety, we also included retrospective, observational studies with more than 30 subjects. Two authors abstracted data and determined risk of bias using a recognised rating system of trial quality.

Results: Four randomised controlled trials (RCTs) and two additional retrospective, observational studies were identified and included in the review. Three RCTs of BFRs for students with examination anxiety, and one RCT of BFRs for children with attention-deficit hyperactivity disorder (ADHD) showed no overall benefit in comparison to placebo. Due to the number and quality of the studies the strength of the evidence is low or very low. We did not find any controlled prospective studies regarding the efficacy of BFRs for pain. Only four of the six studies included for safety explicitly reported adverse events.

Conclusion: Most of the available evidence regarding the efficacy and safety of BFRs has a high risk of bias. We conclude that, based on the reported adverse events in these six trials, BFRs are probably safe. Few controlled prospective trials of BFRs for psychological problems and pain exist. Our analysis of the four controlled trials of BFRs for examination anxiety and ADHD indicates that there is no evidence of benefit compared with a placebo intervention. 